\title{
Synthesis and styrene copolymerization of novel bromo, chloro, methoxy, and methyl ring-disubstituted isobutyl phenylcyanoacrylates
}

Adriana M. Fricano, Lily L. Boland, Kelly A. Campbell, Nim Yan Chan, Jared A. Curry, Anh Dam, Jack Q. Gabrish, Clare E. Gordon, Megan E. Gross, Sara M. Rocus, William S. Schjerven and Gregory B. Kharas

DePaul University, Chemistry and Biochemistry Department, 1110 West Belden Avenue, Chicago, IL 60614-3214

*Contact: E-mail gkharas@ depaul.edu

\begin{abstract}
Novel ring-disubstituted isobutyl phenylcyanoacrylates, $\mathrm{RPhCH}=\mathrm{C}(\mathrm{CN}) \mathrm{CO}_{2} \mathrm{CH}_{2} \mathrm{CH}\left(\mathrm{CH}_{3}\right)_{2}$ (where $\mathrm{R}$ is 2-bromo-3-methoxy, 2-bromo-5-methoxy, 3-bromo-4-methoxy, 5-bromo-2methoxy, 2-bromo-4-methyl, 2-chloro-3-methoxy, 3-chloro-4-methoxy, 3-chloro-4-methyl) were synthesized by the piperidine catalyzed Knoevenagel condensation of ringdisubstituted benzaldehydes and isobutyl cyanoacetate and characterized by $\mathrm{CHN}$ analysis, IR, ${ }^{1} \mathrm{H}$ and ${ }^{13} \mathrm{C}$ NMR. The acrylates were copolymerized with styrene in solution with radical initiation $(\mathrm{ABCN})$ at $70^{\circ} \mathrm{C}$. The compositions of the copolymers were between 19.6 and $32.6 \mathrm{~mol} \%$ of acrylates. Weight-average molecular masses of the copolymers were 41.3 - 54.5kD. Decomposition of the copolymers in nitrogen occurred in two steps, first in the
\end{abstract}


$248-500^{\circ} \mathrm{C}$ range with residue $(1.5-6.1 \% \mathrm{wt})$, which then decomposed in the $500-800^{\circ} \mathrm{C}$ range.

\section{Introduction}

Bromo and methoxy ring-disubstituted ethyl phenylcyanoacrylate (PCA) was used in synthesis of thiazacridine derivatives as anticancer agents against breast and hematopoietic neoplastic cells [1]; in synthesis of a novel thiazolidinedione and evaluation of its modulatory effect on IFN- $\gamma$, IL-6, IL-17A, and IL-22 production in PBMCs from rheumatoid arthritis patients [2]; in synthesis and anti-inflammatory activity of new arylidene-thiazolidine-2,4-diones as PPAR $\gamma$ ligands [3]; in preparation of 2,4thiazolidinedione derivatives having hypoglycemic activity [4]; in synthesis and study of biological activity of novel acridinylidene and benzylidene thiazolidinediones [5]; in synthesis and study of anti-inflammatory activity of new thiazolidine-2,4-diones, 4thioxothiazolidinones, and 2-thioxoimidazolidinones [6]; in synthesis and studyschistosomicidal activity of new substituted thioxo-imidazolidine compounds [7]; in studies of behavior of Schistosoma Mansoni adult worms maintained in vitro towards imidazolidinone derivatives [8]; in synthesis of 1,3,5-trisubstituted-2thioxoimidazolidinones [9]; in studies of stereochemistry of arylidenecyanoacetic acids and arylarylideneacetonitriles [10]. Bromo and methyl ring-disubstituted ethyl PCA were involved in preparation of 1-heteroaryl-2-phenylcyclopropanes used for allosteric modulation of nicotinic acetylcholine receptors [11]. Chloro and methoxy ring-disubstituted ethyl PCA was involved in application of ethyl $p$-methoxy cinnamate and its derivatives in 
the maintenance of stem cell self-renewal and pluripotency $[12,13]$, as well as in synthesis of 2-methoxy-5-chloro-, 3-chloro-4-methoxy-, and 3,4-dichlorophenylsuccinic acids [14]. Chloro and methyl isopropyl PCA was used in tunable Cinchona-Based thioureas-catalysed asymmetric epoxidation to synthetically important glycidic ester derivatives [15]. Earlier we have reported synthesis and styrene copolymerization a number of bromo, chloro, methoxy, and methyl ring-disubstituted PCAs, as methyl [16], ethyl [17], propyl [18, 19], isopropyl [20], butyl [21], isobutyl [22], methoxyethyl [23], and octyl [24].

Thus, in continuation of our investigation of novel PCA compounds we have prepared bromo, chloro, methoxy, and methyl ring-disubstituted isobutyl PCA, $\mathrm{RPhCH}=\mathrm{C}(\mathrm{CN}) \mathrm{CO}_{2} \mathrm{CH}_{2} \mathrm{CH}\left(\mathrm{CH}_{3}\right)_{2}$, where $\mathrm{R}$ is 2-bromo-3-methoxy, 2-bromo-5-methoxy, 3-bromo-4-methoxy, 5-bromo-2-methoxy, 2-bromo-4-methyl, 2-chloro-3-methoxy, 3chloro-4-methoxy, 3-chloro-4-methyl, and explored the feasibility of their copolymerization with styrene. To the best of our knowledge, there have been no reports on either synthesis of these compounds, nor their copolymerization with styrene [25].

\section{Experimental}

2-Bromo-3-methoxy, 2-bromo-5-methoxy, 3-bromo-4-methoxy, 5-bromo-2-methoxy, 2bromo-4-methyl, 2-chloro-3-methoxy, 3-chloro-4-methoxy, 3-chloro-4methylbenzaldehydes, isobutyl cyanoacetate, piperidine, styrene, 1,1'azobis(cyclohexanecarbonitrile) (ABCN), and toluene supplied from Sigma-Aldrich Co., were used as received. Instrumentation is described in [26]. 


\section{Results and discussion}

\subsection{Synthesis and characterization of isobutyl phenylcyanoacrylates}

All isobutyl phenylcyanoacrylates (IPCA) compounds were synthesized by Knoevenagel condensation [27] of appropriate benzaldehydes with isobutyl cyanoacetate, catalyzed by base, piperidine (Scheme 1).

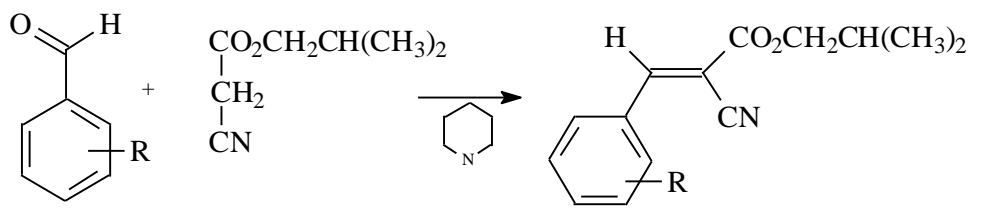

Scheme 1. Synthesis of isobutyl phenylcyanoacrylates, where R is 2-bromo-3-methoxy, 2bromo-5-methoxy, 3-bromo-4-methoxy, 5-bromo-2-methoxy, 2-bromo-4-methyl, 2-chloro3-methoxy, 3-chloro-4-methoxy, 3-chloro-4-methyl.

The preparation procedure was essentially the same for all the monomers. In a typical synthesis, equimolar amounts of isobutyl cyanoacetate and an appropriate benzaldehyde were mixed in equimolar ratio in a $20 \mathrm{~mL}$ vial. A few drops of piperidine were added with stirring. The reactions were allowed to proceed $48 \mathrm{hrs}$. at rt. The product of the reaction was isolated by filtration and purified by crystallization from 2-propanol. The condensation reaction proceeded smoothly, yielding products, which were purified by conventional techniques. Melting points of the compounds in crystalline state were measured by DSC.

The compounds were characterized by IR, ${ }^{1} \mathrm{H}$ and ${ }^{13} \mathrm{C}$ NMR spectroscopies. No stereochemical analysis of the novel ring-substituted IPCA was performed since no stereoisomers ( $E$ or/and $Z$ ) of known configuration were available. 


\subsubsection{Isobutyl 2-bromo-3-methoxyphenylcyanoacrylate}

Yield: $94.2 \%$; ${ }^{1} \mathrm{H}$ NMR: $\delta 8.7$ (s, $\left.1 \mathrm{H}, \mathrm{CH}=\right), 7.8-6.7(3 \mathrm{H}, \mathrm{Ph}), 4.1\left(\mathrm{~d}, 2 \mathrm{H}, \mathrm{CH}_{2}\right), 4.0(\mathrm{~s}$, $\left.3 \mathrm{H}, \mathrm{OCH}_{3}\right), 2.1(\mathrm{~m}, 1 \mathrm{H}, \mathrm{CH}), 0.9\left(\mathrm{~d}, 6 \mathrm{H}, \mathrm{CH}_{3}\right) ;{ }^{13} \mathrm{C} \mathrm{NMR}: \delta 163(\mathrm{C}=\mathrm{O}), 153(\mathrm{HC}=)$, 149, 137, 128, $127(\mathrm{Ph}), 115(\mathrm{CN}), 103(\mathrm{C}=), 52\left(\mathrm{OCH}_{3}\right), 28(\mathrm{CH}), 18\left(\mathrm{CH}_{3}\right) ; \mathrm{IR}:\left(\mathrm{cm}^{-1}\right)$ 3224-2834 (m, C-H), 2233 (m, CN), 1747 (s, C=O), 1647 (s, C=C), 1271 (s, C-O-CH3), 810 (s, C-H out of plane). Anal. calcd. for $\mathrm{C}_{15} \mathrm{H}_{16} \mathrm{BrNO}_{3}: \mathrm{C}, 53.27 ; \mathrm{H}, 4.77 ; \mathrm{N}, 4.14$; Found: C, 50.73; H, 4.34; N, 4.11.

\subsubsection{Isobutyl 2-bromo-5-methoxyphenylcyanoacrylate}

Yield 92\%; mp 75.2 ${ }^{\circ} \mathrm{C} ;{ }^{1} \mathrm{H}$ NMR $\delta 8.6(\mathrm{~s}, 1 \mathrm{H}, \mathrm{CH}=), 7.8-7.0(3 \mathrm{H}, \mathrm{Ph}), 4.1\left(\mathrm{~d}, 2 \mathrm{H}, \mathrm{CH}_{2}\right)$, $3.9\left(\mathrm{~s}, 3 \mathrm{H}, \mathrm{OCH}_{3}\right), 2.1(\mathrm{~m}, 1 \mathrm{H}, \mathrm{CH}), 1.0\left(\mathrm{~d}, 6 \mathrm{H}, \mathrm{CH}_{3}\right) ;{ }^{13} \mathrm{C} \mathrm{NMR} \delta 163(\mathrm{C}=\mathrm{O}), 154$ $(\mathrm{HC}=), 133,132,128,127,126,112(\mathrm{Ph}), 116(\mathrm{CN}), 103(\mathrm{C}=),\left(\mathrm{CH}_{2}\right), 53\left(\mathrm{OCH}_{3}\right), 26$ $(\mathrm{CH}), 16\left(\mathrm{CH}_{3}\right)_{2} ; \mathrm{IR}\left(\mathrm{cm}^{-1}\right): 3250-2750(\mathrm{~m}, \mathrm{C}-\mathrm{H}), 2230(\mathrm{~m}, \mathrm{CN}), 1728(\mathrm{~s}, \mathrm{C}=\mathrm{O}), 1607$ $(\mathrm{C}=\mathrm{C}), 1228\left(\mathrm{~s}, \mathrm{C}-\mathrm{O}-\mathrm{CH}_{3}\right), 823,763$ (s, C-H out of plane). Anal. Calcd. for $\mathrm{C}_{15} \mathrm{H}_{16} \mathrm{BrNO}_{3}$ : C, 53.27; H, 4.77; N, 4.14; Found: C, 53.46; H, 4.81; N, 4.87.

\subsubsection{Isobutyl 3-bromo-4-methoxyphenylcyanoacrylate}

Yield 83\%; mp $135.1^{\circ} \mathrm{C} ;{ }^{1} \mathrm{H}$ NMR $\delta 8.1(\mathrm{~s}, 1 \mathrm{H}, \mathrm{CH}=), 7.3,7.0(\mathrm{~d}, 3 \mathrm{H}, \mathrm{Ph}), 4.1(\mathrm{~d}, 2 \mathrm{H}$, $\left.\mathrm{CH}_{2}\right), 4.0\left(\mathrm{~s}, 3 \mathrm{H}, \mathrm{OCH}_{3}\right), 2.2(\mathrm{~m}, 1 \mathrm{H}, \mathrm{CH}), 1.0\left(\mathrm{~d}, 6 \mathrm{H}, \mathrm{CH}_{3}\right) ;{ }^{13} \mathrm{C} \mathrm{NMR} \delta 163(\mathrm{C}=\mathrm{O}), 153$ $(\mathrm{HC}=), 159,149,136,126,111(\mathrm{Ph}), 116(\mathrm{CN}), 101(\mathrm{C}=), 73\left(\mathrm{CH}_{2}\right), 57\left(\mathrm{OCH}_{3}\right), 28$ (CH), $19\left(\mathrm{CH}_{3}\right)$; IR $\left(\mathrm{cm}^{-1}\right)$ : $2962(\mathrm{~m}, \mathrm{C}-\mathrm{H}), 2222(\mathrm{~m}, \mathrm{CN}), 1717$ (s, C=O), $1587(\mathrm{C}=\mathrm{C})$, 1265 (s, C-O-CH 3 ), 822, 750 (s, C-H out of plane). Anal. Calcd. for $\mathrm{C}_{15} \mathrm{H}_{16} \mathrm{BrNO}_{3}$ : C, 53.27; H, 4.77; N, 4.14; Found: C, 53.18; H, 4.85; N, 4.19. 


\subsubsection{Isobutyl 5-bromo-2-methoxyphenylcyanoacrylate}

Yield 76\%; mp $103.4^{\circ} \mathrm{C} ;{ }^{1} \mathrm{H}$ NMR $\delta 8.6(\mathrm{~s}, 1 \mathrm{H}, \mathrm{CH}=)$, 8.3, 7.6, 6.9 (s, 3H, Ph), 4.1 (d, $\left.2 \mathrm{H}, \mathrm{CH}_{2}\right), 3.9\left(\mathrm{~s}, 3 \mathrm{H}, \mathrm{OCH}_{3}\right), 2.1(\mathrm{~m}, 1 \mathrm{H}, \mathrm{CH}), 1.0\left(\mathrm{~d}, 6 \mathrm{H}, \mathrm{CH}_{3}\right) ;{ }^{13} \mathrm{C} \mathrm{NMR} \delta 162(\mathrm{C}=\mathrm{O})$, $158(\mathrm{HC}=), 148,137,132,122,113(\mathrm{Ph}), 115(\mathrm{CN}), 104(\mathrm{C}=), 73\left(\mathrm{CH}_{2}\right), 56\left(\mathrm{OCH}_{3}\right), 28$ (CH), $19\left(\mathrm{CH}_{3}\right)$; IR (cm-1): 3020-2811 (m, C-H), 2224 (m, CN), 1724 (s, C=O), 1603 (s, $\mathrm{C}=\mathrm{C}$ ), 1248 (s, C-O-CH 3 ), 812, 758 (s, C-H out of plane). Anal. Calcd. for $\mathrm{C}_{15} \mathrm{H}_{16} \mathrm{BrNO}_{3}$ : C, 53.27; H, 4.77; N, 4.14; Found: C, 53.44; H, 4.87; N, 4.21.

\subsubsection{Isobutyl 2-bromo-4-methylphenylcyanoacrylate}

Yield 94\%; mp $63.0^{\circ} \mathrm{C} ;{ }^{1} \mathrm{H}$ NMR $\delta 8.6(\mathrm{~s}, 1 \mathrm{H}, \mathrm{CH}=), 8.1,7.5,7.2(\mathrm{~s}, 3 \mathrm{H}, \mathrm{Ph}), 4.1(\mathrm{~s}, 2 \mathrm{H}$, $\left.\mathrm{CH}_{2}\right), 2.4\left(\mathrm{~s}, 3 \mathrm{H}, \mathrm{CH}_{3}\right), 2.1(\mathrm{~m}, 1 \mathrm{H}, \mathrm{CH}), 1.0\left(\mathrm{~d}, 6 \mathrm{H}, \mathrm{CH}_{3}\right) ;{ }^{13} \mathrm{C} \mathrm{NMR} \delta 162(\mathrm{C}=\mathrm{O}), 153$ $(\mathrm{HC}=), 134,130,127,125,113(\mathrm{Ph}), 115(\mathrm{CN}), 105(\mathrm{C}=), 73\left(\mathrm{CH}_{2}\right), 28(\mathrm{CH}), 21$ $\left(\mathrm{PhCH}_{3}\right), 20\left(\mathrm{CH}_{3}\right)_{2}$; IR $\left(\mathrm{cm}^{-1}\right): 2904$ (m, C-H), 2225 (m, CN), 1728 (s, C=O), 1599 (s, $\mathrm{C}=\mathrm{C}$ ), 1293 (s, C-O-CH 3 ), 825, 752 (s, C-H out of plane). Anal. Calcd. for $\mathrm{C}_{15} \mathrm{H}_{16} \mathrm{BrNO}_{2}$ : C, 55.92; H, 5.01; N, 4.35; Found: C, 55.76; H, 5.08; N, 4.69.

\subsubsection{Isobutyl 2-chloro-3-methoxyphenylcyanoacrylate}

Yield 92\%; mp 84.6 ${ }^{\circ} \mathrm{C} ;{ }^{1} \mathrm{H}$ NMR $\delta 8.7$ (s, $\left.1 \mathrm{H}, \mathrm{CH}=\right), 7.8-7.0(\mathrm{~m}, 3 \mathrm{H}, \mathrm{Ph}), 4.2(\mathrm{~d}, 2 \mathrm{H}$, $\left.\mathrm{CH}_{2}\right), 3.9\left(\mathrm{~s}, 3 \mathrm{H}, \mathrm{OCH}_{3}\right), 2.1(\mathrm{~m}, 1 \mathrm{H}, \mathrm{CH}), 1.1\left(\mathrm{~d}, 6 \mathrm{H}, \mathrm{CH}_{3}\right) ;{ }^{13} \mathrm{C} \mathrm{NMR} \delta 162(\mathrm{C}=\mathrm{O}), 156$ $(\mathrm{HC}=), 152,131,127,124,122,114(\mathrm{Ph}), 116(\mathrm{CN}), 107(\mathrm{C}=), 73\left(\mathrm{CH}_{2}\right), 57\left(\mathrm{OCH}_{3}\right), 28$ (CH), $19\left(\mathrm{CH}_{3}\right)_{2}$; IR $\left(\mathrm{cm}^{-1}\right)$ : 2964 (m, C-H), $2226(\mathrm{~m}, \mathrm{CN}), 1728$ (s, C=O), 1610 (s, C=C), 1269 (s, C-O-CH 3 ), 852, 752 (s, C-H out of plane). Anal. Calcd. for $\mathrm{C}_{15} \mathrm{H}_{16} \mathrm{ClNO}_{3}$ : C, 61.33; H, 5.49; N, 4.77; Found: C, 62.10; H, 5.43; N, 4.84. 


\subsubsection{Isobutyl 3-chloro-4-methoxyphenylcyanoacrylate}

Yield 87\%; mp $120.5^{\circ} \mathrm{C} ;{ }^{1} \mathrm{H}$ NMR $\delta 8.1$ (s, $\left.1 \mathrm{H}, \mathrm{CH}=\right), 8.0-7.0(\mathrm{~m}, 3 \mathrm{H}, \mathrm{Ph}), 4.1(\mathrm{~s}, 2 \mathrm{H}$, $\left.\mathrm{CH}_{2}\right), 4.0\left(\mathrm{~s}, 3 \mathrm{H}, \mathrm{OCH}_{3}\right), 2.1(\mathrm{~m}, 1 \mathrm{H}, \mathrm{CH}), 1.0\left(\mathrm{~d}, 6 \mathrm{H},\left(\mathrm{CH}_{3}\right)_{2} ;{ }^{13} \mathrm{C} \mathrm{NMR} \delta 164(\mathrm{C}=\mathrm{O})\right.$, $154(\mathrm{HC}=), 153,132,127,126(\mathrm{Ph}), 115(\mathrm{CN}), 102(\mathrm{C}=), 71\left(\mathrm{CH}_{2}\right), 56\left(\mathrm{CH}_{3} \mathrm{O}\right), 28(\mathrm{CH})$, $19\left(\mathrm{CH}_{3}\right)_{2} ; \mathrm{IR}\left(\mathrm{cm}^{-1}\right): 2970(\mathrm{~m}, \mathrm{C}-\mathrm{H}), 2225(\mathrm{~m}, \mathrm{CN}), 1717$ (s, C=O), 1601 (s, C=C), 1263 (s, C-O-CH 3 ), 802, 752 (s, C-H out of plane). Anal. Calcd. for $\mathrm{C}_{15} \mathrm{H}_{16} \mathrm{ClNO}_{3}: \mathrm{C}, 61.33 ; \mathrm{H}$, 5.49; N, 4.77; Found: C, 59.81; H, 5.61; N, 4.91.

\subsubsection{Isobutyl 3-chloro-4-methylphenylcyanoacrylate}

Yield 86\%; mp $115.0^{\circ} \mathrm{C} ;{ }^{1} \mathrm{H}$ NMR $\delta 8.2(\mathrm{~s}, 1 \mathrm{H}, \mathrm{CH}=), 8.0-7.3(\mathrm{~m}, 3 \mathrm{H}, \mathrm{Ph}), 4.1(\mathrm{~d}, 2 \mathrm{H}$, $\left.\mathrm{CH}_{2}\right), 2.5\left(\mathrm{~s}, 2 \mathrm{H}, \mathrm{CH}_{3}\right), 2.1(\mathrm{~m}, 1 \mathrm{H}, \mathrm{CH}), 1.0\left(\mathrm{~d}, 6 \mathrm{H}, \mathrm{CH}_{3}\right) ;{ }^{13} \mathrm{C} \mathrm{NMR} \delta 162(\mathrm{C}=\mathrm{O}), 153$ $(\mathrm{HC}=), 142$ 135, 132, 131, $129(\mathrm{Ph}), 115(\mathrm{CN}), 103(\mathrm{C}=), 72\left(\mathrm{CH}_{2}\right), 28(\mathrm{CH}), 21\left(\mathrm{CH}_{3}\right)$, $19\left(\mathrm{CH}_{3}\right)_{2}$; IR ( $\left.\mathrm{cm}^{-1}\right): 2964$ (m, C-H), $2224(\mathrm{~m}, \mathrm{CN}), 1717$ (s, C=O), 1618 (s, C=C), 1248 (s, C-O-CH 3 ), 812, 758 (s, C-H out of plane). Anal. Calcd. for $\mathrm{C}_{15} \mathrm{H}_{16} \mathrm{ClNO}_{2}: \mathrm{C}, 64.97 ; \mathrm{H}$, 5.81; N, 5.04; Found: C, 65.74; H, 5.74; N, 5.15.

\subsection{Synthesis and characterization of styrene - IPCA copolymers}

Copolymers of the styrene (ST) and the IPCA compounds, P(ST-co-IPCA) were prepared in $25-\mathrm{mL}$ glass screw cap vials at ST/IPCA $=3(\mathrm{~mol})$ the monomer feed using $0.12 \mathrm{~mol} / \mathrm{L}$ of $\mathrm{ABCN}$ at an overall monomer concentration $2.44 \mathrm{~mol} / \mathrm{L}$ in $10 \mathrm{~mL}$ of toluene. The copolymerization was conducted at $70^{\circ} \mathrm{C}$. After a predetermined time, the mixture was cooled to room temperature, and precipitated dropwise in methanol. The novel synthesized IPCA compounds copolymerized readily with ST under free-radical 
conditions (Scheme 2) forming white flaky precipitates when their solutions were poured into methanol. The conversion of the copolymers was kept between 10 and $20 \%$ to minimize compositional drift (Table 1). The composition of the copolymers was determined based on the nitrogen content.

Table 1. Copolymerization of isobutyl phenylcyanoacrylates with styrene.

\begin{tabular}{|c|c|c|c|c|c|c|c|c|c|}
\hline & & & & & & $T G A$ & & & \\
\hline $\mathrm{R}$ & $\begin{array}{l}\text { Yield }^{\mathrm{a}} \\
(\mathrm{wt} \%)\end{array}$ & $\begin{array}{l}\mathrm{N} \\
(\mathrm{wt} \%)\end{array}$ & $\begin{array}{l}\mathrm{m}_{2} \text { in } \\
\text { copol. } \\
(\mathrm{mol} \%)\end{array}$ & $\begin{array}{l}\mathrm{Mw}_{\mathrm{W}} \\
(\mathrm{kD})\end{array}$ & $\begin{array}{l}\mathrm{T}_{\mathrm{g}} \\
\left({ }^{\circ} \mathrm{C}\right)\end{array}$ & $\begin{array}{l}\text { Onset of } \\
\text { decomp. } \\
\left({ }^{\circ} \mathrm{C}\right)\end{array}$ & $\begin{array}{l}10 \\
\mathrm{wt} \% \\
\text { loss } \\
\left({ }^{\circ} \mathrm{C}\right)\end{array}$ & $\begin{array}{l}50 \\
\mathrm{wt} \% \\
\text { loss } \\
\left({ }^{\circ} \mathrm{C}\right)\end{array}$ & $\begin{array}{l}\text { Residue } \\
\text { wt } \%\end{array}$ \\
\hline $2-\mathrm{Br}-4-\mathrm{CH}_{3} \mathrm{O}$ & 12.3 & 1.83 & 19.6 & 54.5 & 127 & 265 & 299 & 345 & 2.4 \\
\hline $2-\mathrm{Br}-5-\mathrm{CH}_{3} \mathrm{O}$ & 16.1 & 2.53 & 32.6 & 47.4 & 146 & 270 & 304 & 342 & 1.7 \\
\hline $3-\mathrm{Br}-4-\mathrm{CH}_{3} \mathrm{O}$ & 11.1 & 2.21 & 26.0 & 51.2 & 142 & 268 & 306 & 338 & 3.1 \\
\hline $5-\mathrm{Br}-2-\mathrm{CH}_{3} \mathrm{O}$ & 12.6 & 2.02 & 22.7 & 45.2 & 147 & 274 & 300 & 334 & 1.5 \\
\hline $2-\mathrm{Br}-4-\mathrm{CH}_{3}$ & 11.3 & 2.24 & 25.6 & 42.7 & 142 & 269 & 308 & 348 & 5.1 \\
\hline 2-Cl-3- $\mathrm{CH}_{3} \mathrm{O}$ & 11.2 & 2.56 & 29.1 & 43.6 & 139 & 272 & 312 & 344 & 2.8 \\
\hline $3-\mathrm{Cl}-4-\mathrm{CH}_{3} \mathrm{O}$ & 12.5 & 2.28 & 24.5 & 41.3 & 137 & 274 & 305 & 341 & 1.8 \\
\hline $3-\mathrm{Cl}-4-\mathrm{CH}_{3}$ & 14.1 & 2.34 & 24.5 & 42.6 & 130 & 248 & 311 & 352 & 6.1 \\
\hline
\end{tabular}

Copolymerization (Scheme 1) of ST and the ring-disubstituted ICPA resulted in formation of copolymers (Table 1) with weight-average molecular masses 41.3 - 54.5kD. Nitrogen elemental analysis showed that between 19.6 and $32.6 \mathrm{~mol} \%$ of IPCA is present in the 
copolymers, which is indicative of relatively high reactivity of the IPCA monomers towards ST radical. Since IPCA monomers do not homopolymerize, the most likely structure of the copolymers would be isolated IPCA monomer $(y=1)$ units alternating with short ST sequences $(x>1)($ Scheme 2$)$.

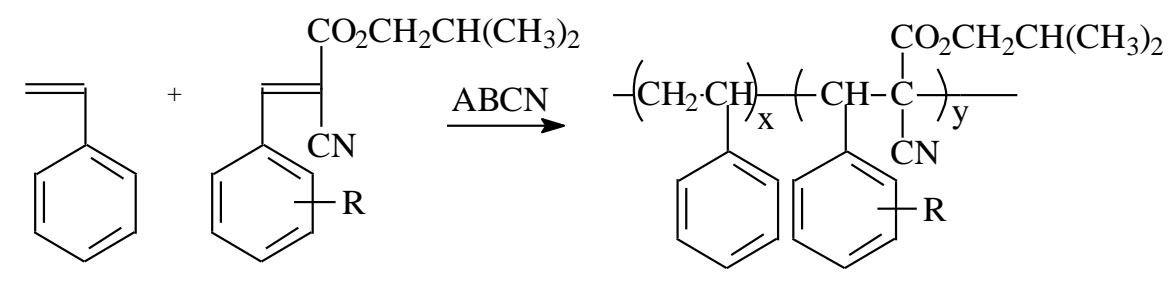

Scheme 2. Copolymerization of ST and the ring-substituted isobutyl phenylcyanoacrylates, $\mathrm{RPhCH}=\mathrm{C}(\mathrm{CN}) \mathrm{CO}_{2} \mathrm{CH}_{2} \mathrm{CH}\left(\mathrm{CH}_{3}\right)_{2}, \mathrm{R}=$ 2-bromo-3-methoxy, 2bromo-5-methoxy, 3-bromo-4-methoxy, 5-bromo-2-methoxy, 2-bromo-4-methyl, 2-chloro3-methoxy, 3-chloro-4-methoxy, 3-chloro-4-methyl.

The copolymers prepared in the present work are all soluble in ethyl acetate, THF, DMF and $\mathrm{CHCl}_{3}$ and insoluble in methanol, ethyl ether, and petroleum ether. Thermal behavior was studied by DSC and TGA. All the copolymers were amorphous and show no crystalline DSC endotherm on repeated heating and cooling cycles. Table 1 shows glass transition values for the ST-ICPA copolymers prepared in this work with no correlation to the size and position of the ICPA ring substitution apparently due to non-uniform composition, monomer unit distribution, and/or molecular weight and MWD. A single $T \mathrm{~g}$ was observed for all the copolymers with values $127-147^{\circ} \mathrm{C}$. Information on thermal stability of the copolymers was obtained from TGA (Table 1). Decomposition of the copolymers 
in nitrogen occurred in two steps, first in the $248-500^{\circ} \mathrm{C}$ range with residue $(1.5-6.1 \%$ wt), which then decomposed in the $500-800^{\circ} \mathrm{C}$ range. The decomposition products were not analyzed in this study, and the mechanism has yet to be investigated.

\section{Conclusions}

Novel ring-disubstituted isobutyl phenylcyanoacrylates were prepared and copolymerized with styrene. The compositions of the copolymers were calculated from nitrogen analysis. Decomposition of the copolymers in nitrogen occurred in two steps, first in the 248$500^{\circ} \mathrm{C}$ range with residue $\left(1.5-6.1 \%\right.$ wt), which then decomposed in the $500-800^{\circ} \mathrm{C}$ range.

\section{Acknowledgments}

The authors are grateful to acknowledge that the project was partly supported by Chicago Society of Coating Technology (CSCT).

\section{References}

1. Synthesis of thiazacridine derivatives as anticancer agents against breast and hematopoietic neoplastic cells. Galdino-Pitta, Marina R.; Pereira, Michelly C.; Quirino, Michael W. L.; Rego, Moacyr J. B. M.; Lima, Maria do Carmo A.; Pitta, Maira G. R.; Pitta, Ivan R. Latin American Journal of Pharmacy (2017), 36(1), 59-67.

2. Synthesis of a novel thiazolidinedione and evaluation of its modulatory effect on IFN- $\gamma$, IL-6, IL-17A, and IL-22 production in PBMCs from rheumatoid arthritis patients. Ferreira da Rocha, Laurindo, Jr.; Rego, Moacyr Jesus Barreto de Melo; Cavalcanti, 
Mariana Brayner; Pereira, Michelly Cristiny; Pitta, Marina Galdino da Rocha; Santana de Oliveira, Priscilla Stela; Goncalves, Sayonara Maria Calado; Duarte, Angela Luzia Branco Pinto; Alves de Lima, Maria do Carmo; Pitta, Ivan da Rocha; et al. BioMed Research International (2013), 926060, 9 pp.

3. Synthesis and anti-inflammatory activity of new arylidene-thiazolidine-2,4-diones as PPAR $\gamma$ ligands. Barros, Cleiton Diniz; Amato, Angelica Amorim; Bento de Oliveira, Tiago; Iannini, Karime Bicas Rocha; Lauro da Silva, Anekecia; Goncalves da Silva, Teresinha; Leite, Elisa Soares; Hernandes, Marcelo Zaldini; Alves de Lima, Maria do Carmo; Galdino, Suely Lins; et al. Bioorganic \& Medicinal Chemistry (2010), 18(11), 3805-3811.

4. Preparation of 2,4-thiazolidinedione derivatives having hypoglycemic activity. Pitta, Ivan da Rocha; Galdino, Suely Lins; Alves de Lima, Maria do Carmo. Braz. Pedido PI (2007), BR 2006001826 A 20071127.

5. Synthesis and Biological Activity of Novel Acridinylidene and Benzylidene thiazolidinediones. Mourao, R. H.; Silva, T. G.; Soares, A. L. M.; Vieira, E. S.; Santos, J. N.; Lima, M. C. A.; Lima, V. L. M.; Galdino, S. L.; Barbe, J.; Pitta, I. R. European Journal of Medicinal Chemistry (2005), 40(11), 1129-1133.

6. Synthesis and anti-inflammatory activity of new thiazolidine-2,4-diones, 4thioxothiazolidinones and 2-thioxoimidazolidinones. Santos, L. C.; Uchoa, F. T.; Canas, A. R. P. A.; Sousa, I. A.; Moura, R. O.; Lima, M. C. A.; Galdino, S. L.; Pitta, I. R.; Barbe, J. Heterocyclic Communications (2005), 11(2), 121-128. 
7. Synthesis and schistosomicidal activity of new substituted thioxo-imidazolidine compounds. Albuquerque, M. C. P. A.; Silva, T. G.; Pitta, M. G. R.; Silva, A. C. A.; Silva, P. G.; Malagueno, E.; Santana, J. V.; Wanderley, A. G.; Lima, M. C. A.; Galdino, S. L.; et al. Pharmazie (2005), 60(1), 13-17.

8. Behavior of Schistosoma mansoni adult worms maintained in vitro towards imidazolidinone derivatives. Oliveira, Silvania M.; Albuquerque, Monica C. P. A.; Pitta, Maira G. R.; Malagueno, Elisabeth; Santana, Jose V.; Lima, Maria do Carmo A.; Pitta, Ivan R.; Galdino, Suely L. Acta Farmaceutica Bonaerense (2004), 23(3), 343348.

9. A novel way of synthesis of 1,3,5-trisubstituted-2-thioxoimidazolidinones. Brandao, S. S. F.; Andrade, A. M. C.; Pereira, D. T. M.; Barbosa Filho, J. M.; Lima, M. C. A.; Galdino, S. L.; Pitta, I. R.; Barbe, J. Heterocyclic Communications (2004), 10(1), 9-14. 10. Stereochemistry of arylidenecyanoacetic acids and arylarylideneacetonitriles. Baker, Wilson; Howes, C. S. Journal of the Chemical Society (1953), 119-24.

11. 1-Heteroaryl-2-phenylcyclopropanes as allosteric modulators of nicotinic acetylcholine receptors and their preparation. Crowley, Brendan M.; Campbell, Brian T.; Duffy, Joseph L.; Greshock, Thomas J.; Guiadeen, Deodial G.; Harvey, Andrew John; Huff, Belinda C.; Leavitt, Kenneth J.; Rada, Vanessa L.; Sanders, John M.; et al. PCT Int. Appl. (2017), WO 2017165256 A1 20170928.

12. Application of ethyl p-methoxycinnamate and derivatives in maintaining self-renewal and pluripotency of stem cells. Bao, Yongli; Li, Yuxin; Qin, Hongshuang. PCT Int. Appl. (2016), WO 2016119085 A1 20160804. 
13. Application of ethyl p-methoxy cinnamate and its derivatives in the maintenance of stem cell self-renewal and pluripotency. Bao, Yongli; Li, Yuxin; Qin, Hongshuang. Faming Zhuanli Shenqing (2015), CN 104726399 A 20150624.

14. Substituted dicarboxylic acids. I. Synthesis of 2-methoxy-5-chloro-, 3-chloro-4methoxy-, and 3,4-dichlorophenylsuccinic acids. Genge, D. K.; Trivedi, J. J. Journal of the Indian Chemical Society (1957), 34, 915-16.

15. Tunable Cinchona-Based Thioureas-Catalysed Asymmetric Epoxidation to Synthetically Important Glycidic Ester Derivatives. Meninno, Sara; Zullo, Luca; Overgaard, Jacob; Lattanzi, Alessandra. Advanced Synthesis \& Catalysis (2017), 359(6), 913-918.

16. Novel Copolymers of Styrene. 12. Halogen Ring-Substituted Methyl 2-Cyano-3-Phenyl2-Propenoates. C.E. Karver, E.S. Molina, E.T. Economos, J.C. Fuentes, S.M. Kaitson, S. Kogan, G. Levi, J.K. Marcus, S. Mei, S.C. Reinsalu, K. Rico, M.M. Zuzek, G.B. Kharas. J. Macromol. Sci. A51(2) 101-105 (2014).

17. Novel Copolymers of Styrene. 8. Haloalkyl Ring-substituted Ethyl 2-Cyano-3-phenyl-2propenoates. G.B. Kharas, B.M. Fitzpatrick, M.C. Francis, C. Gallardo, J.L. Gehle, G.M. Giovannini, M.E. Giovannini, J.K. Jerzewski, S.L. LaFary, L.N. Lessor, D.E. Macias. J. Macromol. Sci. A50 (4) 370-374 (2013).

18. Novel Copolymers of Styrene. 7. Some Ring-Disubstituted Propyl 2-Cyano-3-Phenyl-2Propenoates. G.B. Kharas, V.A. Sloan-Lyon, M. Cieszynski, J.D.A. Manuel, S.L. Mei, J.B. Mubang Jr, P.E. Skorseth, Kaila E. Smith, N.M. Tassone, E. Tolberd, and D. Z.W. Yan. J. Macromol. Sci.A53(12) 729-733 (2016). 
19. Novel Copolymers of Styrene. 10. Bromo and Chloro Ring-Disubstituted Propyl 2Cyano-3-Phenyl-2-Propenoates. G.B. Kharas, J. Bates, A.T. Boyd, J.N. Burke, J.L. Crosby, S.B. Sundquist, C.C. Zoleta, A.K. Zurek, and P.L. Bromby II. J. Macromol. Sci. A54(6) 372-375 (2017).

20. Synthesis and styrene copolymerization of novel halogen, alkoxy, and alkyl ringdisubstituted isopropyl 2-cyano-3-phenyl-2-propenoates. K.M. Hussain, R.M. Breault, N.J. Caporusso, C.J. Cu, K.J. Decker, T.K. Gill, E.K. Gray, C.J. Homsi, M.F. Kanji, R.M. Stone, J.L. Zepeda, S.M. Rocus, W.S. Schjerven, G.B. Kharas. ChemRxiv (04.10.2019). https://doi.org/10.26434/chemrxiv.9932393

21. Novel Copolymers of Styrene. 7. Some Ring-Disubstituted Butyl 2-Cyano-3-Phenyl-2-

Propenoates. G.B. Kharas, H. Feng, I.S. Shouib, C. Tong, A. Tsang, D. Velazquez, A.C. Williamson, E.B. Yokana, and A.M. Zekic. J. Macromol. Sci. A53(1) 1-5 (2016).

22. Synthesis and styrene copolymerization of novel ring-disubstituted isobutyl phenylcyanoacrylates. L.L. Boland, K.A. Campbell, Nim Yan Chan, J.A. Curry, Anh Dam, J.Q. Gabrish, C.E. Gordon, M.E. Gross, S.M. Rocus, W.S. Schjerven, G.B. Kharas. ChemRxiv (17.08.2020). https://doi.org/10.26434/chemrxiv.11401347.v5

23. Synthesis and styrene copolymerization of novel trisubstituted ethylenes: 7. Some ringdisubstituted 2-methoxyethyl phenylcyanoacrylates. Imaad Allahrakha, Vikram Bhagavat, Justin Bodner, Deanna M. Briones, Jacqueline C. Calderon, Nadia Quad, Abdul Rafay, Claire M. Sagartz, Sid Sarfaraz, Adam W.T. Steffeck, Sabah M. Sulaiman, Stephanie L. Taiberg, Skyler M. Thompson, Joanna L. Torres, Sara M. 
Rocus, William S. Schjerven, Gregory B. Kharas. ChemRxiv. Preprint. (26.01.2021) https://doi.org/10.26434/chemrxiv.13262660.v7

24. Synthesis and styrene copolymerization of novel trisubstituted ethylenes: 6. Methyl, halogen and oxy ring-disubstituted octyl phenylcyanoacrylates. Emma J. Clajus, Natalie Cote, Rama Dalloul, Giulia M. DiMarco, Mollie J. Eriksson, Yesenia Garcia, Nathalie A. Gijsbers, Jay H. Kaila, Amina S. Malik, Madeeha I. Mohiuddin, Anaa Mulk, Neil T. Patel, Sara M. Rocus, William S. Schjerven, and Gregory B. Kharas. ChemRxiv. Cambridge Open Engage Version 17 Jul 11, 2021. https://doi.org/10.33774/chemrxiv-2021-k812v-v17

25. SciFinder structure search Feb 232022.

26. Synthesis and styrene copolymerization of novel alkyl ring-substituted isobutyl 2-cyano3-phenyl-2-propenoates. Schjerven, William S.; Groy, Randi; Antonishina, Yekaterina S.; Black, Claire I.; Bolin, Madysen P.; Caniglia, John; Daroach, Deepak P.; Farooqui, Farah T.; Feiz, Yalda; Garcia, Sam; Gasparini, Gabriella; Kharas, Gregory B. Abstracts of Papers, 259th ACS National Meeting \& Exposition, Philadelphia, PA, United States, March 22-26, (2020), POLY-0388.

27. Smith, M. B.; March, J. Addition to Carbon-Hetero Multiple Bonds, In March's Advanced Organic Chemistry, J. Wiley \& Sons: New York, Ch.16, 1225 (2001). 